\title{
Empatía en enfermería y el contexto de la relación enfermero-paciente: consideraciones críticas
}

\section{Nursing empathy and the context of the nurse-patient relationship: critical considerations \\ Empatia em enfermagem e o contexto da relação enfermeiro-paciente: considerações críticas}

\author{
Leandro Felipe Mufatoํㅜㄹ Maria Aparecida Munhoz Gaíva² \\ ${ }^{1}$ Enfermeiro. Mestre em Enfermagem. Doutorando em Enfermagem pelo \\ Programa de Pós-Graduação em Enfermagem da Universidade Federal de Mato \\ Grosso. Professor Assistente da Universidade do Estado de Mato Grosso- \\ UNEMAT, Tangará da Serra, Mato Grosso, Brasil. Correo electrónico: \\ leandro.mufato@gmail.com \\ ${ }^{2}$ Doutora em Enfermagem. Professora do Programa de Pós-Graduação em \\ Enfermagem da Universidade Federal de Mato Grosso, Cuiabá, Mato Grosso, \\ Brasil. Pesquisadora do CNPq. Correo electrónico: mamgaiva@yahoo.com.br \\ Cómo citar este artículo en edición digital: Mufato, L.F. E Munhoz Gaíva, M.A. (2019). Empatia en \\ enfermería y el contexto de la relación enfermero-paciente: consideraciones críticas. Cultura de los \\ Cuidados (Edición digital), 23 (54). Recuperado de http://dx.doi.org/10.14198/cuid.2019.54.06
}

Correspondencia: Rua San Francisco, N.410, Apto.1106, Torre 1. Edifício Garden Shangri-lá. CEP:78070-370. Cuiabá, Mato Grosso

Correo electrónico de contacto: mleandro.mufato@gmail.com Recibido: 20/12/2018; Aceptado: 12/03/2019

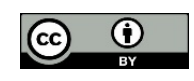

\begin{abstract}
Empathy is an ability to understand the other, fundamental in the nurse-patient relationship. In nursing, Theresa Wiseman formulated her own concepts about empathy from the characteristics of the profession. However, after more than ten years of the empathy concept in nursing formulated by the author, we have to observe the current context of occurrence of this phenomenon: the nurse-patient relationship. In this essay, we have looked at some factors that demonstrate the importance of empathy between nurses and patients, as well as what challenges the
\end{abstract}

profession faces to promote its autonomy and which directly interfere with the way nurses occupy space in relation to their patients and consequently in the development of empathy. Nursing work in wards, the division of labor with technicians and submission of the profession to the modes of production of hospital care are presented as aspects that interfere in the nurse-patient relationship and in empathy. It is concluded that there is a need to resume individualized care actions in the direct relationship with patients, so that the nurse can be seen as a resource, as a source of care for patients. 
Keywords: Empathy, nurse-patient relations, nursing, nursing care, interpersonal relationships.

\section{RESUMO}

A empatia é considerada uma habilidade para compreender o outro, fundamental na relação enfermeiro-paciente. $\mathrm{Na}$ enfermagem, Theresa Wiseman formulou conceitos próprios sobre a empatia a partir das características da profissão. No entanto, depois de mais de dez anos do conceito de empatia em enfermagem formulado pela autora, temos que nos deter sobre o contexto atual de ocorrência deste fenômeno: a relação enfermeiro-paciente. Neste ensaio, lançamos olhares para alguns fatores que demonstram a importância da empatia entre enfermeiros e pacientes, bem como, que desafios a profissão enfrenta para a promoção da sua autonomia e que interferem diretamente no modo como os enfermeiros ocupam o espaço na relação com seus pacientes e, consequentemente, no desenvolvimento da empatia. O trabalho de enfermagem em enfermarias, a divisão do trabalho com técnicos e a submissão da profissão aos modos de produção do cuidado hospitalar apresentam-se como aspectos que interferem na relação enfermeiro-paciente e na empatia. Concluise pela necessidade de retomada de ações de cuidado individualizados na relação direta com os pacientes, de modo que o enfermeiro possa ser visto como um recurso, como uma fonte de cuidados pelos pacientes.

Palavras chave: Empatia, relações enfermeiro-paciente, enfermagem, cuidados de enfermagem, relações interpessoais.

\section{RESUMEN}

La empatía se considera una habilidad para comprender al otro, fundamental en la relación enfermero-paciente. En la enfermería, Theresa Wiseman formuló conceptos propios sobre la empatía a partir de las características de la profesión. Sin embargo, después de más de diez años del concepto de empatía en enfermería formulado por la autora, tenemos que detenernos sobre el contexto actual de incidencia de este fenómeno: la relación enfermero-paciente. En este ensayo, observamos algunos factores que demuestran la importancia de la empatía entre enfermeros y pacientes, también, qué desafíos la profesión enfrenta para la promoción de su autonomía y que interfieren directamente en el modo como los enfermeros ocupan el espacio en la relación con sus pacientes y en el desarrollo de la empatía. El trabajo de enfermería en centros sanitarios, la división del trabajo con técnicos y la sumisión de la profesión a los modos de producción del cuidado hospitalario se presentan como aspectos que interfieren en la relación enfermero-paciente y en la empatía. Se concluye por la necesidad de retomada de acciones de cuidado individualizadas en la relación directa con los pacientes, de modo que el enfermero pueda ser visto como un recurso, como una fuente de cuidados por los pacientes.

Palabras clave: Empatía, relaciones enfermero-paciente, enfermería, cuidados de enfermería, relaciones interpersonales.

\section{INTRODUÇÃO}

A empatia é uma forma de cognição social que nos permite o conhecimento dos outros. Este tema tem ressurgido em diferentes pesquisas e converge para si diversas teorias que explicam sua ocorrência. Esse ressurgimento e crescimento do debate sobre a empatia tem ocorrido no campo da filosofia e sociologia - cognição social, e, 
também, expressivamente no campo da saúde. A onda que trouxe a empatia de modo forte na agenda filosófica e científica ocorreu, em parte, devido à descoberta dos "neurônios espelhos", um conjunto de neurônios motores capazes de serem ativados quando observamos uma ação realizada por outra pessoa, como se fossemos nós que estivéssemos realizando. Sobre esta descoberta se depositou a confirmação empírica de hipóteses filosóficas sobre como compreendemos outra pessoa (Zahavi, 2012).

No campo da saúde é naturalizada a ocorrência de cuidados no contexto de uma relação direta profissional-paciente. Logo, nesta relação a compreensão e conhecimento que se tem do outro é fundamental para o sucesso terapêutico dos cuidados. Especificamente, o cuidado de enfermagem carrega a premissa da relação entre dois sujeitos, um que cuida e outro que necessita de cuidados. O cuidado de enfermagem envolve um "encontro interpessoal com objetivo terapêutico" (Pires, 2009, p. 742), favorecido quando o profissional compreende e conhece as necessidades do paciente, ou seja, favorecido pela empatia.

O debate atual e crescente sobre os relacionamentos profissionais-pacientes no campo da saúde tem sido alavancado em boa parte por pesquisas sobre a empatia entre médicos, enfermeiros e psicólogos com seus pacientes (Reynolds \& Scott, 2000; Wittenberg-Lyles et al. 2012; Birhanu, Tsion, Mirkuzie \& Sudhakar, 2012; Soltner, Giquello, Monrigal-Martin \& Beydon, 2011; Moghaddasian, Dizaji \& Mahmoudi, 2013). Dessa forma, a empatia torna-se um tema que extrapola o campo específico da enfermagem e se torna uma preocupação de várias profissões da saúde, aliás, a psicologia é quem primeiro se ocupou desta temática para finalidades terapêuticas.
Apesar da empatia surgir no início do século XX como conceito no campo da saúde, a enfermagem desenvolveu referências específicas sobre a empatia somente a partir da década de 1960. Tereza Wiseman (2007) estudou a atividade de profissionais da enfermagem, analisando a empatia destes profissionais e formulou seu próprio conceito de empatia com base em pesquisas empíricas. Para essa autora, a empatia em saúde não contemplava as características da profissão enfermagem, que vão muito além dos padrões de consultas empregados pela medicina e psicologia. Sua tipologia da empatia se mostra própria da enfermagem por ter sido empiricamente observada em enfermarias de cuidados oncológicos. Empregaremos as ideias de empatia em enfermagem de Wiseman neste ensaio, como modo de colocar a relevância do tema para a enfermagem.

Os enfermeiros deveriam desenvolver com as pessoas a quem prestam cuidados uma experiência humana significativa que contribua para o seu desenvolvimento (Vieira, 2008). Neste sentido, o relacionamento entre enfermeiro e paciente deve possibilitar a ocorrência da terapêutica própria da enfermagem, e a empatia se releva uma forma do enfermeiro compreender seu paciente, atraindo para a enfermagem a discussão em torno da compreensão social do outro, proposta pelos estudiosos da empatia.

Apesar de ser um encontro intersubjetivo com finalidade terapêutica, o que por si demanda atitudes empáticas do profissional, se nos determos nas características do trabalho da enfermagem na atualidade, somos indagados, pelos olhares estranhos à profissão, e por vezes dos próprios enfermeiros: mas, onde entra a empatia na enfermagem? Parte deste 
questionamento se fundamenta na observação crítica sobre a profissão, que hoje se caracteriza por um trabalho tarefeiro, não individualizado,

desorganizado politicamente, centrado nos aspectos administrativos e de gestão, e, principalmente, pouco conectado com o paciente (Machado, 2010; McQueen, 2000). Se aos poucos nos ocupamos de outras atividades que nos distanciam dos pacientes como podemos discutir a empatia dos enfermeiros?

Disseminou-se um certo senso comum, social e acadêmico, de que o profissional enfermeiro vive situações de trabalho com pouco tempo para o cuidado, sem dedicação na relação direta enfermeiro-paciente, portanto, sem bons níveis de empatia. Este profissional frustrado e pressionado pelo produtivismo, característica marcante dos serviços de saúde, tem demonstrado isto em suas atitudes e comportamentos junto aos pacientes, aumentando ainda mais o distanciamento entre ambos (McQueen, 2000).

Nesse sentido, pretendemos aqui ampliar o espaço de discussão das características da profissão que influenciam diretamente a ocorrência da empatia. No rumo desta problematização, defendemos o argumento de que a enfermagem sempre esteve preocupada em participar da vida das pessoas doentes, de modo a facilitar-lhes a recuperação, além de participar também da vida de pessoas saudáveis para promover sua saúde e prevenir a ocorrência de adoecimentos. Evidencia-se o quanto os enfermeiros somente "são" profissionais do cuidado, se suas ações permitirem o bemestar do outro, se o cuidado de enfermagem se fizer necessário para as pessoas seguirem com seus projetos e motivações na vida, aspectos inalcançáveis se o enfermeiro não se relacionar diretamente com seus pacientes de modo empático, ou seja, sem que o enfermeiro compreenda e conheça seu paciente.

Nos dias atuais, paira sobre nossas cabeças uma densa nuvem de desconhecimento da relevância clínica e social do cuidado de enfermagem (Machado, 2010) e, do valor de sua mais nobre característica, a relação ininterrupta com as pessoas em situações de saúde e de doença. Diante deste cenário, questionamos: $\mathrm{O}$ que aconteceu com a enfermagem que a fez perder espaço na relação direta com o paciente e tornou a empatia algo estranho à profissão em alguns cenários de atuação?

Assim, este ensaio teórico procura refletir sobre a profissão de enfermagem como uma atividade voltada para as pessoas doentes, imbricada, portanto, na relação e na compreensão empática de seus pacientes. Procura, também, elencar as inquietantes, provocantes e desafiadoras características do desenvolvimento profissional, que levaram o enfermeiro a se distanciar de seus pacientes e perder o foco de sua valorização política, organização profissional e de sua própria contribuição para a estima social que deveria ser atribuída ao cuidado de enfermagem.

\section{A relação enfermeiro-paciente na centralidade do desenvolvimento da profissão de enfermagem}

O ser humano sempre esteve conceituado pela enfermagem como foco de sua atuação. Em todas as teorias de enfermagem o homem/ser humano aparece sendo conceituado, e suas necessidades para desenvolver-se plenamente constituem a espinha dorsal da profissão e da ciência enfermagem.

No desenvolvimento profissional, a relação enfermeiro-paciente foi a tônica nas definições de "o que seria a enfermagem". O 
ser humano necessitando de cuidados sempre esteve presente nas propostas teóricas de enfermagem. Para exemplificar o quanto a relação enfermeiro-paciente se fez importante no pensamento de teoristas da enfermagem, podemos trazer a ideia de Joyce Travelbee. Em Travelbee, vemos a relação enfermeiro-paciente como a essência do propósito da enfermagem e trata-se de uma relação pessoa-pessoa, estabelecida por meio da empatia (Leopardi, 1999). Nota-se que teoristas de enfermagem usam o termo empatia no estabelecimento da relação enfermeiro-paciente, sem contudo, se preocupar em conceituar o que viria a ser a empatia para a enfermagem. É notável, também que empatia e simpatia foram usados na enfermagem como sinônimos de características desejáveis ao cuidado, sem que fossem problematizados e melhor definidos até a década de 1970.

Ao conceituar a relação enfermeiropaciente como a essência da enfermagem, observa-se o quanto esta relação se fez significativa para a profissão, marcadamente até a década de 1960. A partir de 1960, no caso brasileiro, ocorre um incremento do ensino voltado para formar líderes e administradores em enfermagem, um dos fatores que afastaram os enfermeiros de seus pacientes (Machado, 2010). Este e outros fatores serão explorados mais adiante.

Após o desenvolvimento desta primeira escola de pensamento na enfermagem, que privilegiava a relação entre profissionais e pacientes, vieram outras, algumas focando nas necessidades dos pacientes, outras nos aspectos cuidativos em relação aos curativos (Vieira, 2008). Independente da escola de pensamento, a enfermagem sempre se viu precisando conceituar teoricamente quem é este ser humano que necessita de seus cuidados, além de, e talvez por causa de, lidar com uma longa jornada de atividades junto de seus pacientes na prática, o que a diferenciava dos aspectos "consultoriais" da medicina e da psicologia.

O trabalho em enfermagem envolve uma relação entre sujeitos (Pires, 2009). Esta autora coloca que o cuidador e o sujeito cuidado possuem expectativas e interesses que podem aproximar-se, potencializando o cuidado de si e do outro, ou distanciar-se, gerando conflitos. A história recente da enfermagem brasileira depara-se com esta mesma lógica de pensamento, a de que a enfermagem participa da trajetória de vida do sujeito enfermo com o objetivo de tornálo mais autônomo na sua vida (Schoeller, 2010).

Neste sentido, trajetórias de vida dos sujeitos e sua autonomia, são abordagens para o cuidado de enfermagem que vão muito além de lidar com um corpo doente. Nessa complexidade do objeto de trabalho, compreender o paciente tornou-se essencial para a eficácia dos cuidados de enfermagem, colocando a empatia como um requisito e atributo do profissional de enfermagem. Mesmo assim, somente mais tarde pode-se discutir de modo mais específico o que seria a empatia para a enfermagem.

As mudanças que tornam a empatia cada vez mais necessária para os cuidados de enfermagem também ocorrem por meio das necessidades em saúde da população. Estas se deslocam dos prejuízos e injúrias ao corpo físico no início do século XX para outras dimensões mais complexas, como a vida diante das vulnerabilidades sociais, os desafios psicológicos de acompanhar a sociedade atual em suas transformações, a abertura do campo da saúde mental, lidando com adoecimentos da mente e não do corpo, as doenças crônicas que exigem um cuidado contínuo, o envelhecimento populacional, entre outros. Nesse 
desenvolvimento, a enfermagem ampliou seus cenários de atuação, levando consigo a estreita relação com os pacientes e a necessidade de compreendê-los, agora não mais como um corpo doente, mas como um sujeito social, uma pessoa. Como diria Zahavi (2014), é necessário olharmos o outro como uma mente corporificada, e não como uma mente e um corpo, separado como dois opostos. Esta compreensão se faz cada vez mais necessária para a enfermagem.

$\mathrm{Na}$ história recente da enfermagem, Schoeller (2010) à coloca categoricamente como a profissão do cuidado, e afirma que o cuidado terapêutico pressupõe

conhecimento, mas não somente o conhecimento intelectual e científico, como também o conhecimento do outro [...] Este conhecimento deve contemplar as dimensões do biológico, do espiritual, do afetivo, do econômico e do social e cultural, na tentativa, [...] de conhecermos o mais profundamente possível esse outro ser e seu momento de vida (p. 248).

Observamos que conhecer o outro, seu paciente, se faz em parte para contemplar outras dimensões que não a biológica. Quando elevamos o cuidado de enfermagem para além do biológico, necessitando conhecer mais das necessidades em saúde dos pacientes, saber conhecer o outro se torna essencial na profissão, ou seja, a empatia passa a ser essencial.

\section{A empatia em enfermagem proposta por Theresa Wiseman: uma forma de compreender o paciente}

O termo empathy foi introduzido no idioma inglês aproximadamente em 1902. O termo deriva da expressão alemã einfuhling, usada pelo filósofo Robert Vischer e empregada por Theodor Lipps para designar o estado de "estar dentro" de um sentimento (infeeling). Os psicólogos e psicoterapeutas foram os primeiros a dominar o termo na área da saúde (López, Filippetti \& Richaud, 2014; White, 1997). Carl Rogers (1957, p. 95) afirma que a empatia é "a noção de estar no mundo do cliente como se fosse seu próprio mundo, sem perder a característica do "como se fosse".

Até 1950 era comum o termo simpatia ser utilizado pela enfermagem para tratar da relação enfermeiro-paciente. Influenciados pelo psicólogo Carl Rogers, os primeiros estudiosos da empatia na enfermagem são da década de 1960 e 1970.

A confusão entre simpatia e empatia na enfermagem foi clarificada por Wiseman (1996), que esclarece que na simpatia nós percebemos a experiência de outra pessoa e imaginamos como nós estaríamos ao vivenciar esta experiência. Na empatia, nós percebemos a experiência de outra pessoa e imaginamos como ela vivencia esta experiência. Em outras palavras, a simpatia fala dos próprios valores, crenças, conhecimento e sentimentos, enquanto que a empatia trata dos valores, crenças, conhecimento e sentimentos de outra pessoa.

A autora Wiseman (1996) que realizou uma análise conceitual e pesquisas empíricas sobre a empatia em enfermagem, afirma que a empatia prescindiria de alguns atributos sem os quais ela não poderia ocorrer: 1) ver o mundo como uma outra pessoa o vê; 2) não julgamento; 3) entendimento dos sentimentos dos outros; e, 4) comunicação deste entendimento.

Neste sentido, a interação e a escuta é algo que antecede a ocorrência da empatia. A escuta seria necessária de ambos os lados da relação profissional-paciente, para que o profissional possa entender os sentimentos do paciente, e para que o paciente possa, do 
outro lado, ter certeza da interação e do entendimento do profissional sobre seus sentimentos (Wiseman, 1996). A necessidade humana básica de ser compreendido seria o primeiro alcance como consequência da empatia

Após aprofundar suas investigações sobre a empatia de enfermeiros numa enfermaria oncológica, Theresa Wiseman (2007) apresenta uma proposta de conceituação da empatia em enfermagem de modo mais holística, ressaltando o fenômeno da empatia em enfermagem como algo multifacetado e identificado em diferentes formas de expressão. A autora apresenta quatro formas da empatia em enfermagem: A empatia como incidente -demonstra que os enfermeiros são empáticos a partir do contexto que propicia tal ocorrência. A enfermaria, a filosofia da instituição, as crenças e valores dos enfermeiros os colocam a serem empáticos; A empatia como uma forma de conhecer- a empatia melhora o acesso às necessidades dos pacientes (bem como possibilita conhecer as necessidades, crenças, valores e prioridades dos indivíduos); A empatia como um processo demonstra que os enfermeiros que são empáticos com seus pacientes o são de modo processual, várias vezes empático com o mesmo paciente. Isso torna mais fácil continuar empático a cada novo momento, por vezes até tornar-se algo inconscientemente; A empatia como uma forma de ser- demonstra que alguns enfermeiros tomam a empatia como uma forma de fazer a enfermagem. Alguns fatores associados à esta forma de ser do enfermeiro incluem familiaridade e similaridade com o paciente, experiência prévia dos enfermeiros em ser um paciente, boa capacidade de comunicação do paciente e a cronicidade do adoecimento do paciente.

Wiseman (2007) ainda reviu alguns aspectos pontuado anteriormente sobre a empatia, demonstrando que o autoconhecimento ou autoconsciência tem papel importante na expressão da empatia, haja vista que os enfermeiros que desenvolvem habilidades empáticas demonstraram ter consciência de seus pontos fortes e de suas fraquezas, o que influenciou o modo como estes interagiam com os pacientes.

Observamos, no entanto, que o contexto de ocorrência da empatia, ou seja, a relação enfermeiro-paciente, se vê ameaçado. As direções tomadas pela própria profissão influenciam neste sentido. As características da profissão que interferem no processo da construção da empatia, seriam: a fragilidade no desenvolvimento de sua autonomia profissional, o baixo alcance do reconhecimento da utilidade social do trabalho profissional de enfermagem (Pires, 2009). Por vezes, a enfermagem surge como uma profissão desvalorizada em suas atividades e incapaz de se organizar politicamente para ocupar o espaço de atuação junto às pessoas (Machado, 2010). Portanto, na discussão da empatia em enfermagem, precisamos elencar alguns aspectos críticos da profissão que diminuem as oportunidades de encontros diretos com seus pacientes, implicando na diminuição das oportunidades empáticas de cuidado.

\section{O afrouxamento da relação direta enfermeiro-paciente: a crise das oportunidades empáticas}

Acreditamos que a evolução da enfermagem para uma prática profissional despolitizada, desorganizada, burocraticamente institucionalizada e submissa à categoria médica (Machado, 2010), tem profunda relação com o distanciamento entre enfermeiros e seus pacientes. 
A história assegurou que o "modelo hospitalar" da atuação de enfermagem, inspirado por Florence Nightingale, se sobrepusesse aos demais campos de atuação do enfermeiro, sendo difundido da Inglaterra para o restante do mundo (Machado, 2010). Atualmente, no ambiente hospitalar, a relação enfermeiro-paciente é prejudicada pela organização do trabalho em tarefas a serem realizadas; o foco da assistência em problemas físicos; o incentivo em evitar envolvimento emocional com os pacientes; e, a valorização excessiva de um saber especializado em saúde (McQueen, 2000), constitui-se barreiras entre os sujeitos que cuidam e que precisam de cuidado.

As atividades de enfermagem associadas ao hospital e ao ensino, trouxeram entre outras consequências: a institucionalização da prática de enfermagem submissa à prática médica; o excesso de ocupação com atividades ligadas à terapêutica e produtividade médica, bem como atividades tidas como domésticas, tais como "fiscalizar portaria, lavanderia, hidráulica e elétrica da estrutura dos serviços de saúde atividades confundidas com 'Administração em enfermagem'” (Machado, 2010, p. 342).

A enfermagem, hoje, é submissa a hierarquia hospitalar, como consequência, há priorização das atividades técnicas e desvalorização dos papeis cuidativos e educativos do enfermeiro. A autonomia dos enfermeiros tem recaído sobre atividades administrativas e ausente na essência da profissão, no processo de cuidar e no cuidado (Bueno \& Queiroz, 2006), minguando a atuação dos enfermeiros no âmbito da relação com seus pacientes, e como consequência, diminuindo a expressão da empatia entre ambos. Consideramos que a dimensão administrativa-gerencial é uma dimensão do processo de cuidar (Pires, 2009), porém, ponderamos que talvez seu peso na balança das atividades de enfermagem esteja contribuindo para o menor valor das demais dimensões, e tornando escasso o contexto das relações diretas entre enfermeiros e seus pacientes.

Além disso, a enfermagem se vê preocupada com tarefas demasiadas objetivas e desconectadas de questões emocionais que são próprias das relações com os pacientes, como aferir pressão arterial, temperatura corporal, frequências cardíacas e respiratórias e registro das características das eliminações orgânicas. Essas atividades por si não propiciam uma relação terapêutica, entendida como aquela que permite o conhecimento das necessidades do paciente por meio da empatia.

A separação das atividades de enfermagem entre nurses e ladies, no início da enfermagem moderna, pode ser interpretado como desvalorização das atividades de cuidado, ao destinar o cuidado para as nurses (mulheres com moral duvidosa e baixa instrução) e o saber e poder sobre o cuidado para as ladies (mulheres instruídas de alta classe social). Neste processo, houve, talvez, o nascimento de um dos primeiros fatores intrínsecos que influenciam de modo prejudicial a relação direta enfermeiros-pacientes, colocando entre eles um outro ator social, o técnico de enfermagem. As nurses tornaram-se os atuais técnicos e auxiliares de enfermagem, enquanto as ladies, as enfermeiras (Machado, 2010).

Outro passo dado pela enfermagem para fora do contexto da relação direta enfermeiro-paciente foi devido à um empurrão da ciência e sua associação ao modo de produção capitalista. A partir do século XX e com o passar do tempo, as tecnologias em saúde, as descobertas do 
campo da cirurgia e os novos tratamentos em situações de emergência, compunham o desenvolvimento de modernas tecnologias e conhecimento médico, ofuscando o desenvolvimento dos cuidados de enfermagem na saúde (Vieira, 2008) e o seu valor social. Os aspectos médicos nos cuidados em saúde e toda a tecnologia em desenvolvimento levaram o tratamento dos doentes a centrar-se na cura, e não no processo para a cura. Neste contexto, saber operar equipamentos complexos e modernos torna-se fonte de poder no campo da saúde, acima do saber compreender o outro, tido como um conhecimento de menor valor.

Assistimos, assim, ao desenvolvimento do saber em saúde centrado na doença e na cura, tão hegemônico no campo da saúde, a ponto de que toda subjetividade e identidade social e histórica dos pacientes ser enquadrada aos interesses médicos de cura e de culto ao corpo. Contudo, é justamente nesta subjetividade, nesta identidade social, cultural e histórica que estão os modos do homem responder às situações de adoecimento, sobre o qual a enfermagem atuaria. Este, seria o conteúdo que a compreensão do outro por meio da empatia poderia dar ao enfermeiro.

A falta das ciências do campo das humanidades na formação do enfermeiro implica em deixar de fora deste processo aspectos relacionais e o desenvolvimento de uma terapêutica própria da enfermagem, menos ligada ao saber médico hegemônico. O desenvolvimento das ciências ligadas ao corpo pesou no desenvolvimento da enfermagem o suficiente para ocupar boa parte da formação dos enfermeiros, não deixando espaço para as humanidades do sujeito que necessita de cuidado (Pires, 2009). Em consequência, o profissional formado neste contexto acaba preterindo a execução de atividades de contato direto com o paciente, priorizando ações burocráticas e de gerenciamento, afastandose da essência da profissão (Ramos, Mesquita, Galvão \& Enders, 2013).

Assim, a enfermagem em seu desenvolvimento profissional, tomou uma direção que a afastou de ações diretas com seus pacientes. A medida que se afasta desse locus de atuação, afasta-se de sua identidade profissional e acaba convencendo-se de que a profissão é a exaustiva e interminável execução de tarefas ligadas a outros interesses, como aos interesses médicos e dos serviços de saúde, em detrimento dos interesses da pessoa cuidada e do desenvolvimento de como podemos compreende-las em suas necessidades em saúde, em suas trajetórias de vida, ou seja, de como podemos ser empáticos com os pacientes.

Esse afastamento é o que conduz o questionamento de como a empatia pode ser expressa e mantida na enfermagem? Se os enfermeiros estão tão atarefados com a administração de equipes e serviços de saúde, a empatia com o paciente se expressa de que forma para a enfermagem? A empatia observada por Theresa Wiseman nas enfermarias oncológicas, pode se aplicar a toda enfermagem?

\section{CONSIDERAÇÕES FINAIS}

No contexto do cuidado de enfermagem há numerosos desafios a serem superados para que a relação enfermeiro-paciente torne-se o foco e a essência da enfermagem (novamente?) e que a empatia possa ser estabelecida como realmente fundamental à profissão.

Diante das necessidades de saúde atuais, é imperativo que o cuidado tome a pessoa como foco, sobre a qual recaia os maiores 
benefícios das terapêuticas utilizadas. A enfermagem se vê enfrentando inúmeros obstáculos e desafios para a construção dessa identidade profissional e de uma ciência que consiga responder à dimensão humana. A relação enfermeiro-paciente foi a fundação da enfermagem e o contexto de atuação dos enfermeiros no início da profissão, o que aparentemente não se manteve no decorrer do desenvolvimento profissional, que se voltou para as atividades não ligadas diretamente aos pacientes, mas aos serviços de saúde e por vezes, aos interesses médicos.

A tese que nos propusemos a defender é de que faz-se necessário retomarmos este contexto de atuação: a proximidade com as pessoas, seus projetos de saúde e como podemos contribuir com estes projetos como profissionais preparados e empoderados de uma terapêutica própria de enfermagem, na relação direta com os pacientes, conhecendo-os por meio da empatia. A empatia ocorre de várias formas para os enfermeiros, quando estes atuam diretamente com seus pacientes, como bem observou Thereza Wiseman. O que mostra que há um campo de conhecimento que é próprio da enfermagem e que pode ser mais e melhor explorado: a empatia como forma de conhecer as necessidades em saúde. Porém, o alcance das ideias de Wiseman sobre a empatia, parece não corresponder a todo cenário de atuação de enfermagem, principalmente quando observamos o que autores colocam como características da profissão no cenário brasileiro.

Ocorre que, os enfermeiros estão saindo do contexto da relação direta com o paciente e do desenvolvimento de saberes que podem contribuir nesta relação, como a empatia. Ao saírem deste contexto estão perdendo-se em meio aos interesses de uma sociedade que valoriza o mensurável, em detrimento do sensível e invisível nas relações terapêuticas. Caminham em direção às ciências biológicas e matematizadas hegemônicas na saúde, em detrimento das investigações que exaltam os significados do estar doente e necessitar de cuidados nas dimensões individual, social e política. Quando assim o fazem, demonstram o quanto se distanciam de oportunidades de conhecer o paciente (o outro) por meio da empatia, o que aponta para uma direção contrária às necessidades atuais nos cuidados em saúde: ser empático.

\section{REFERÊNCIAS}

- Birhanu, Z., Tsion, A., Mirkuzie, W. \& Sudhakar, M. (2012). Predictors of perceived empathy among patients visiting primary health-care centers in central Ethiopia. International Society for Quality in Health Care, 24(2), 161-168. DOI: 10.1093/intqhe/mzs001.

- Bueno, F. M. G. \& Queiroz, M. S. O enfermeiro e a construção da autonomia profissional no processo de cuidar. Revista Brasileira de Enfermagem, 59(2), 222-277. DOI: 10.1590/S0034-71672006000200019.

- Leopardi, M. T. (1999). Teorias em enfermagem: Instrumentos para a prática. Florianópolis, Brasil: Ed. Papa-Livros.

- López, M. B., Filippetti, V. A. \& Richaud, M. C. (2014). Empatía: desde la percepción automática hasta los procesos controlados. Avances en Psicología Latinoamericana, 32(1), 37-51. DOI: dx.doi.org/10.12804/apl32.1.2014.03.

- Machado, W. C. A. (2010). A enfermagem moderna. In Geovanini, T., Moreira, A., Dornelles, S. \& Machado W. C. A. História da enfermagem: Versões e interpretações (pp. 335-347). Rio de Janeiro, Brasil: Revinter.

- McQueen, A. (2000). Nurse-patient relationships and partnership in hospital care. Journal of Clinical Nursing, 9, 723-731. DOI: 10.1046/j.13652702.2000.00424.x.

- Moghaddasian, S., Dizaji, S. L. \& Mahmoudi, M. (2013). Nurses empathy and family needs in the Intensive Care Units. Journal of caring sciences, 2(3), 197-201. DOI: 10.5681/jcs.2013.024.

- Pires, D. (2009). A enfermagem enquanto disciplina, profissão e trabalho. Revista Brasileira de Enfermagem, 62(5), 739-44.

- Ramos, D. K. R., Mesquita, S. K. C., Galvão, M. C. B \& 
Enders, B. C. (2013). Paradigmas da saúde e a (des)valorização do cuidado em enfermagem. Enfermagem em Foco, 4(1), 41-44. DOI: 10.21675/2357707X.2013.v4.n1.501.

- Reynolds, W. J. \& Scott, B. S. (2000). Do nurses and other professional helpers normally display much empathy? Journal of advanced nursing, 31(1), 226-234. DOI: $10.1046 / j .1365-2648.2000 .01242 . x$.

- Rogers, C. R. (1957). The necessary and sufficient conditions of therapeutic personality change. Journal of consulting and clinical psychology. 21, 95-103. Recuperado

de $<\underline{\text { https://app.shoreline.edu/dchris/psych236/Docume }}$ nts/Rogers.pdf $>$.

- Schoeller, S. D. (2010). A nossa história recente - a enfermagem brasileira de 1988 a 2002. In Geovanini, T., Moreira, A., Dornelles, S. \& Machado W. C. A. História da enfermagem: versões e interpretações (pp. 247-275). Rio de Janeiro, Brasil: Revinter.

- Soltner, C., Giquello, J. Á., Monrigal-Martin, C. \& Beydon L. (2011). Continuous care and empathic anaesthesiologist attitude in the preoperative period: impact on patient anxiety and satisfaction. British journal of anaesthesia, 106(5), 680-686. DOI: 10.1093/bja/aer034.

- Vieira, M. (2008). Ser enfermeiro: da compaixão à proficiência. Lisboa, Portugal: Universidade Católica
Editora

- White, S. J. (1997). Empathy: a literature review and concept analysis. Journal of Clinical Nursing. 6, 253257.

- Wiseman, T. (1996). A concept analysis of empathy. Journal of advanced nursing. 23, 1162-1167.

- Wiseman, T. (2007). Toward a holistic conceptualization os empathy for nursing practice. Advances in Nursing Science, 30(3), e61-e72. DOI: 10.1097/01.ANS.0000286630.00011.e3.

- Wittenberg-Lyles, E., Debra, P. O., Demiris, G., Rankin, A., Shaufield, S. \& Kruse. R. L. (2012). Conveying empathy to hospice family caregivers: team responses to caregiver empathic communication. Patient education and counseling, 89, 31-37. DOI: 10.1016/j.pec.2012.04.004.

- Zahavi, D. (2012). Empathy and mirroing: Husserl and Gallese. In R. Breeur \& U. Melle (Eds.), Life, subjectivity $\mathcal{E}$ art: Essas in honor of Rudolf Bernet. (pp. 217-254). Phaenomenologia 201. London, Ingland: Springer Science.

- Zahavi, D. (2014). Empathy and other-direct intencionality. Topoi, 33:129-42. DOI: 10.1007/s11245013-9197-4. 